\title{
Health system strengthening and hypertension management in China
}

\author{
Kehui Huang, Yu Ting Song, Yong Huan He and Xing Lin Feng ${ }^{*}$ (D)
}

\begin{abstract}
Background: Non-communicable diseases are the leading causes of global burden of diseases, and hypertension is one of the most important risk factors. Hypertension prevalence doubled in China in the past decade and affects more than 300 million Chinese people. In the review we systematically searched peer-reviewed publications that link health system level factors with hypertension management in China and provide the current knowledge on how to improve a country's health system to manage the hypertension epidemic.

Methods: A framework was developed to guide the review. The database of PubMed, CNKI were systematically searched from inception to April 13, 2016. Two authors independently screened the searched results for inclusion, conducted data extraction and appraised the quality of studies. Key findings were described according to the framework.

Findings: Five hundred seventy-two publications were identified, where 11 articles were left according to the inclusion and exclusion criteria. The study periods range from 2010 to 2015. All about 11 researches linked health system factors to the outcome of hypertension management. And the outcomes were just focused on the awareness, treatment and control of hypertension but not hypertension incidence. One study is about the role of health system governance, investigating the performance of different organized community health care centers; three studies were about health financing comparing differences in insurance coverage; three studies were about health information practicing the hypertension guidelines of China or the $\mathrm{WHO}$, and the rest three about mechanisms of health service delivery. No researches were identified about physical resources for health and human resources for health.
\end{abstract}

Conclusions: Hypertension prevalence has been rising rapidly in China and the management of hypertension in China is a detection problem rather than treatment problem. Limited evidence shows the positive effect of health system factors on hypertension management and joint efforts from health system and epidemiological researchers are warranted to extend knowledge in this area.

Keywords: Health system, Hypertension management, China

\section{Background}

Non-communicable diseases (NCDs)-including cardiovascular diseases (CVDs), cancer, diabetes, and chronic respiratory diseases-are the leading causes of global burden of diseases [1,2], and are considered as "one of the major challenges for sustainable development in the 21st century" [3]. As one of the most important risk factors of CVDs, the pandemic of hypertension has become a global cause for concern [4]. With a prevalence rate of $40 \%$ [1], it leads to $55 \%$ of the global CVDs mortality burden and $7 \%$ DALYs [5]. Hypertension could be managed well with

\footnotetext{
* Correspondence: fxl@bjmu.edu.cn

School of Public Health, Peking University, Xueyuan road 38\#, Beijing 100191, People's Republic of China
}

low-tech, cost-effective and feasibly implemented primary care interventions, even in low resource settings $[6,7]$. However, the proportions of hypertensive patients under appropriate control varies globally, and uncontrolled hypertension contribute to two-thirds of all strokes and half of all coronary diseases [8].

Confronted with the world crisis of NCDs, previous efforts were largely made in building innovative technologies and improving guidelines in researches and clinical practices $[9,10]$. Only until recently, attentions have been drawn to the strengthening of a nations' health system around the principle of universal health coverage [3]. According to the World Health Organization (WHO), efforts should be harmonized in strengthening a 
country's governance structure in health, health financing, human resource for health, health information, health service delivery, and medicines and technologies from a system perspective in order to provide universal essential health care [11]. However, paucity of evidence was generated to answer the "how" question, particularly for the low-and middle-income countries (LMICs). For example, a recent systematic review conducted in the year 2013 identified 53 studies that analyzed the effects of health system strengthening efforts on hypertension awareness, treatment and control. Among these, only 11 were conducted in LMICs, where only one study was from China. However, this study did not explore any mechanism of health system strengthening efforts in improving hypertension management [12].

As the largest developing country, China bears a large burden of NCDs. Hypertension prevalence doubled in the past decade and affected around 337 million Chinese people in the year 2010 [13]. As a silent killer, $80 \%$ of the CVDs mortality were estimated to be attributed to hypertension nationally, and more than half of those were premature death [14]. In this review, we systematically searched peer-reviewed publications that link health system level factors with hypertension management in the Peoples' Republic of China. From the perspective of health system strengthening and population health management, we provide the current knowledge from China on how to improve a country's health system to manage the hypertension epidemic.

\section{Methods}

Building on the WHO's health system monitoring and evaluation framework, and the six building blocks framework $[15,16]$, we developed a conceptual framework that illustrates how health system factors influence the outcome and impact of hypertension management (Fig. 1). The framework divides the system level inputs into six domains, namely health system governance, physical resources for health, health financing, human resources for health, health information, and health service delivery. We refers health system governance to strategic policy making, system design, effective oversight, coalition-building, regulation, and accountability. We refers physical resources for health to the instruments, equipment and materials to prevent, diagnose, treat, monitor or remit the diseases. For health financing, we use a broad concept incorporating the collection, pooling, and allocation of money to cover the health needs of the people. Thus both the risk pooling and purchasing mechanisms are included. As for human resources for health, we include health workers in different domains of health systems, such as curative, preventive and rehabilitative care services as well as health education, promotion and research. And health information is the information technologies, guidelines which is generated, compiled, analyzed, synthesized and used by health worker for diagnostic or interventional support. Service delivery refers to different strategies and organizational mechanisms to provide quality care. We incorporate all these concepts referring the use of health services in this review: access, availability, utilization, and coverage. We believe that should a country's health system be strengthened in these six domains, it would turn out to deliver adequate and efficient interventions so as to positively affect the outcomes of hypertension management (i.e. the incidence of hypertensive disorders, and the awareness, treatment and control for hypertension), and lead to the mitigation of the burden of diseases attributed to hypertension (including morbidity, mortality, financial risks, quality of life and financial burden ushered in by hypertension). Such a framework was used in this review in developing searching strategy (Table 1), inclusion and exclusion criteria (Table 2) and building constructs for data extraction.

Using the strategy listed in Table 1, we systematically searched the PubMed and China National Knowledge Infrastructure (CNKI). The data were searched from inception to April 13, 2016. Two authors independently screened the search results by title and abstract for eligible studies. And those potentially eligible articles were further screened for inclusion by the full texts. Disagreement were solved by discussions and the inextricable ones were further resolved by the last author. To entrench the data base, references of all the English articles left for full texts analysis were manually screened.

We developed a data extraction form (Additional file 1: Appendix 1), and we built the main constructs for data extraction according to the conceptual framework. Kh Huang, Yt Song and Yh He conducted data extraction under the last author's supervision. The data were structured by study number, first author, title, published year, study year, study design, setting, sample characteristics, health system factors, the outcomes and the impacts of hypertension management. And quality of the studies were appraised according to their study designs.

Conceptually, studies investigating the effect of health system factors on hypertension management were classified and described according to the framework. We then described the key findings based on the extracted data.

\section{Results}

Five hundred seventy-two publications were identified from PubMed searches and 19 from CNKI, where 93 articles were screened out by title and abstract according to the inclusion and exclusion criteria. Full texts of the 93 articles were obtained and assessed for relevance and ten articles were left according to the inclusion and exclusion criteria for this review. All references of the 10 


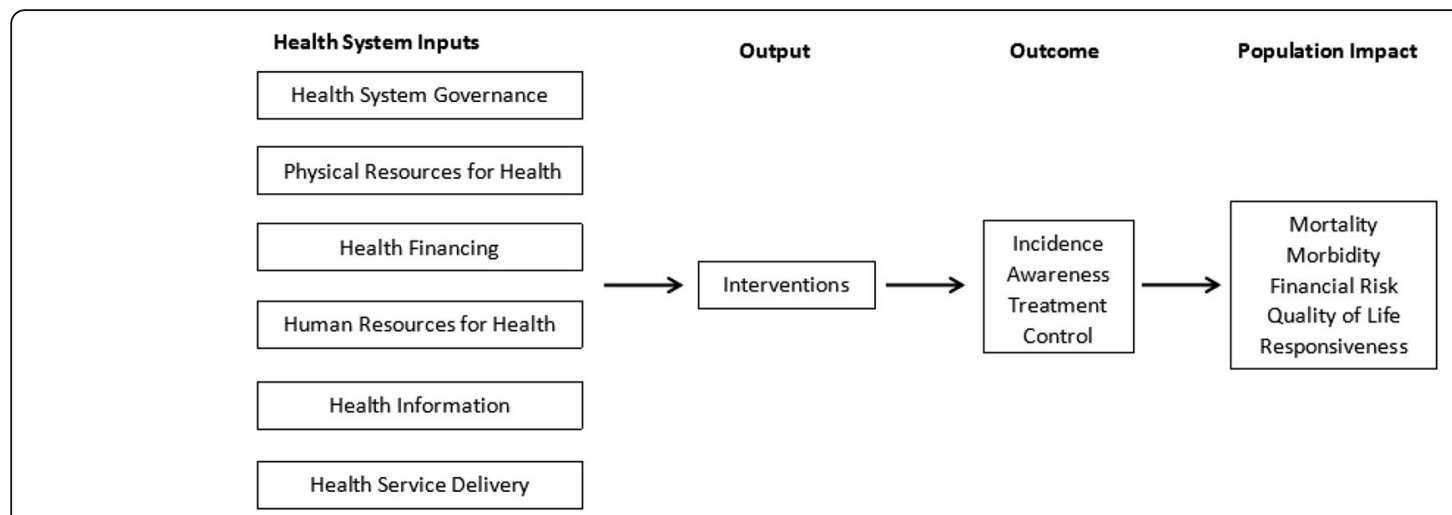

Fig. 1 Conceptual framework for health system strengthening and hypertension management

papers were manually screened and one additional publication were added.

Among the 83 articles excluded, 29 were epidemiological researches purely described the prevalence, awareness, treatment and control of hypertension in China. Twenty-three reported the experiences of community based health promotion programs. These studies were excluded because mechanisms were not disentangled and no efforts were made to link to health system factors. And the other 31 articles were excluded either because the study setting was not in mainland China, or the study was not relative to the outcomes of hypertension management, or the study focused only on mortality, morbidity, quality of life or financial burden attributed to hypertension, or the studies did not provide adequate information.

To describe the current landscape of the outcomes of hypertension management in China, we intentionally

Table 1 Searching strategy

(((((Humans[Mesh]) AND Hypertension[Mesh]) AND China[Tiab]) AND (Case Reports[Publication Type] OR Observational Study[Publication Type] OR Randomized Controlled Trial[Publication Type] OR Comparative Study[Publication Type] OR Evaluation Studies[Publication Type] OR Meta-Analysis[Publication Type] OR Classical Article [Publication Type] OR Corrected and Republished Article[Publication Type] OR Support of Research[Publication Type] OR Health Surveys[Mesh] OR Health Services Research[Mesh])) AND (Delivery of Health Care, Integrated[Mesh] OR Delivery of Health Care[Mesh] OR Health Services Accessibility[Mesh] OR Primary Health Care[Mesh] OR Drug Utilization Review[Mesh] OR Program Evaluation[Mesh] OR Diagnosis[Mesh] OR Self Care[Mesh] OR Health Education[Mesh] OR Equipment and Supplies[Mesh] OR Health Facilities [Mesh] OR Pharmacy[Mesh] OR Pharmaceutical Services[Mesh] OR Health Manpower[Mesh] OR Attitude of Health Personnel[Mesh] OR Health Information Systems[Mesh] OR Health Information Management[Mesh] OR Guideline [Publication Type] OR Healthcare Financing[Mesh] OR Insurance, Health[Mesh] OR Insurance Coverage[Mesh] OR Fees and Charges[Mesh] OR Financing, Government[Mesh] OR Costs and Cost Analysis[Mesh] OR Patient Participation[Mesh] OR Clinical Governance[Mesh] OR Leadership[Mesh] OR Politics[Mesh] OR Health Services Administration[Mesh] OR Health Planning[Mesh] OR Community Health Planning[Mesh] OR Policy[Mesh] OR Health Policy[Mesh] OR Health Priorities[Mesh] OR Health Care Reform[Mesh] OR Health System[All Field] OR National Health Programs[Mesh] OR Social Support[Mesh] OR Social Determinants of Health[Mesh] OR Health Status[Mesh])) extracted information from the 29 epidemiological studies that described the trends in hypertension prevalence, awareness, treatment and control. We combined information from these 29 studies with relevant information from the other 11 papers to describe the current situation for the outcomes of hypertension management in China.

We finally retained a total of 11 researches conducted in China according to our framework. The study periods ranged from the year 2010 to 2015 (Fig. 2). We appraised quality of the researches according to their study designs. Two of the studies included were randomized control trials (RCTs), three were based on quasi-experimental designs, and six were cross-sectional studies. All these researches were conducted in mainland China, with the study population varies from 213 to 29,411 .

Table 2 Inclusion and exclusion criteria

\section{Inclusion criteria}

Studies conducted in Chinese population.

Studies that reported the effect of at least one health system factors on the outcome or impact of hypertension management. Outcomes of hypertension management refers to hypertension awareness, treatment and control. Impact of hypertension management refers to mortality, morbidity, financial risks or quality of life that were attributed to hypertension.

Studies that reported hypertension prevalence, awareness, treatment or control.

Studies that reported any of the following the population impact of hypertension.

\section{Exclusion criteria}

Studies not conducted on humankind.

Studies conducted outside China.

All commentaries, letters and editorials.

Studies merely quantify the effects of personal behavioral or modifiable risk factors on hypertension outcomes.

Studies that only describe the current knowledge of hypertension, i.e awareness, treatment and control.

Studies that merely focus on the population impact of hypertension like mortality, morbidity or financial risk.

Studies that only concentrated on community interventions to improve hypertension management without clearly explications of the mechanisms. 


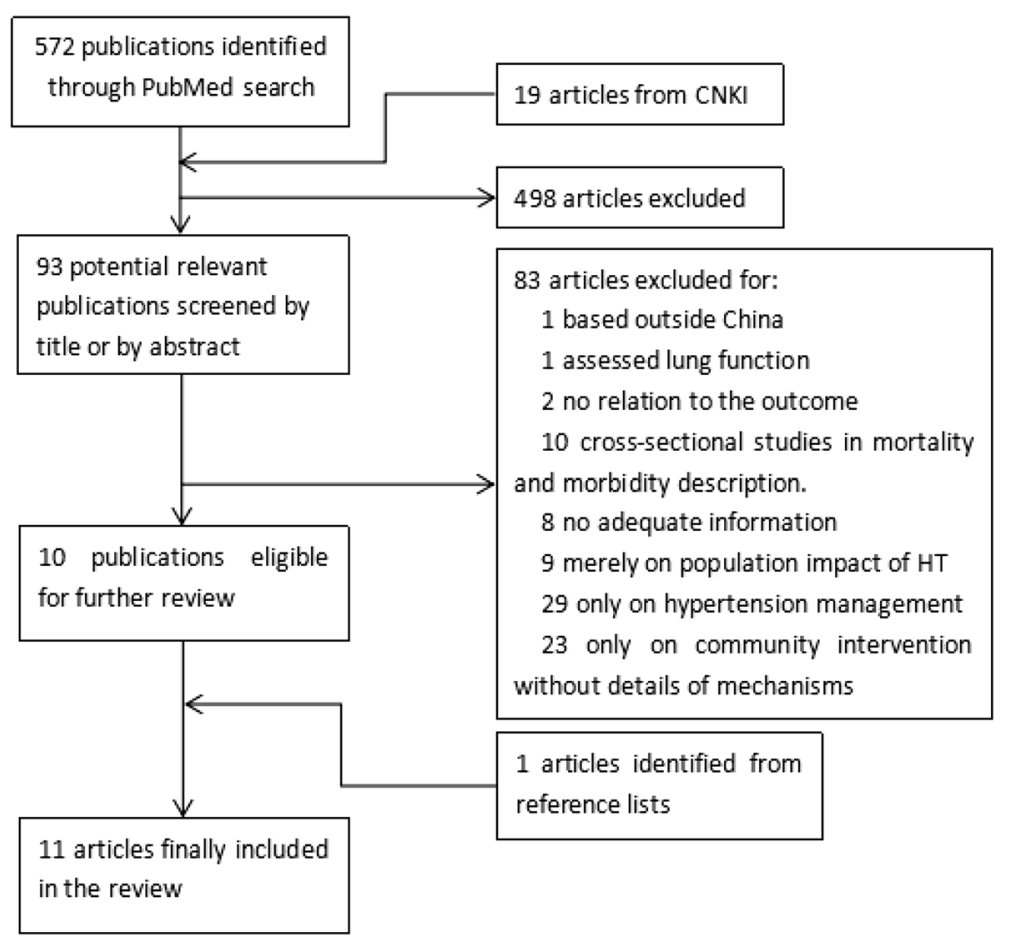

Fig. 2 Flowchart demonstrating literature review procedure. ${ }^{*} \mathrm{CNKl}$, China National Knowledge Infrastructure

\section{The prevalence, awareness, treatment and control of hypertension}

As reported, $27.2 \%$ of the Chinese adults aged 35 to 74. years, representing 129,824,000 persons, had hypertension in 2000 [17]. Whilst 10 years later, based on national data, a prevalence of $40.9 \%$ among people aged 45 years or older was reached [13]. The rapid rising in prevalence seems weird but was corroborated by other sources [13, 17-22]. For example using the WHO's Study on Global Aging and Adult Health (SAGE) data, Basu et.al. reported a prevalence of $39 \%$ in China during 2007-2010 for people no less than 45 years of age [23]. Whilst with the same data, Lloyd-Sherlock et.al reported a prevalence rate of $59.5 \%$ for Chinese people aged no less than 50 years of age [24]. In summary, hypertension awareness, treatment and control were just 33.719 .9 and $12.6 \%$ in 1991 , but increased to $50.0,56.1$ and $36.4 \%$ in 2012. Although improvement were made in improving its management during the past decade, the rates were still unacceptably low, since the screening and routine management of hypertension has been freely provided in China's current health reform since 2009 [22, 24-31]. The project was included in national essential public health service to provide the population with health record establishment and blood pressure monitoring. And as for the hypertensives screened out, it required the primary health center to do at least four follow-ups each year [32]. Furthermore, the effective management of hypertension largely becomes a case identification problem since only half of the Chinese people with hypertension are aware of their disorders, whlist majority of the hypertensive individuals who were aware of their hypertension received antihypertensive medication and most appeared to be under effective control [13].

\section{Health system factors associated with good hypertension management}

Finally 11 most relevant researches investigating the effects of health system factors on the outcomes or impact of hypertension management were retained. All these identified studies just analyzed the effectiveness of health system factors on hypertension awareness, treatment and control. But none of them intended to link health system factors and incidence of hypertension nor the morbidity, mortality, quality of life or financial burden. One was about health system governance investigating the performance of different organized community health care centers, three were about health financing comparing differences in insurance coverage, three were about health information practicing the hypertension guidelines of China or the WHO, and the rest four were about mechanisms of health service delivery. Unfortunately it was not yet studied for the effects of other two health system domains, namely human resources for health and physical resources for health on the outcomes or impact of hypertension management in China (Table 3). 
Table 3 Key findings of the eleven most important studies

\begin{tabular}{|c|c|c|c|c|c|c|}
\hline & Author & Published year & No. and title & Source & Study population & Objective \\
\hline $\begin{array}{l}\text { Health System } \\
\text { Governance }\end{array}$ & Wong et al & 2012 & $\begin{array}{l}\text { Performance Comparison } \\
\text { among the Major Healthcare } \\
\text { Financing Systems in Six Cities } \\
\text { of the Pearl River Delta Region, } \\
\text { Mainland China. }\end{array}$ & PLOS ONE & $\begin{array}{l}1830 \text { patients } \\
\text { of the Pearl } \\
\text { River Delta }\end{array}$ & $\begin{array}{l}\text { Compare the effect } \\
\text { pf hospital-funded, } \\
\text { government-funded } \\
\text { and private-funded } \\
\text { CHCs }\end{array}$ \\
\hline
\end{tabular}

The hypertension treatment rate in hospital-funded community health care centers (CHCs) ranged from
83.1 to $92.1 \%$, which was lower in government83.1 to $92.1 \%$, which was lower in government-
funded CHCs $(70.3 \%$, adjusted OR $0.4695 \%$ Mainland China. $\quad$ CHCS

$\mathrm{Cl} 0.33-0.66)$ and private-funded CHCs (29.9\%, adjusted OR $0.0395 \% \mathrm{Cl} 0.02-0.05$ ); the control rate in hospital-funded CHCs $22.7 \%$, ranged from 20.1 to $28.9 \%$, which was higher in the Government-funded (25.8\%, adjusted OR 1.63 , $95 \%$ Cl 1.16-2.29), lower in the private-funded CHC (8.9\%, adjusted OR $0.1595 \%$ Cl 0.069-0.31).

$\begin{array}{lllll}\begin{array}{l}\text { Health } \quad \text { Guo et al } 2015 \\ \text { Financing }\end{array} & \begin{array}{l}\text { The dynamics of hypertension } \\ \text { prevalence, awareness, treatment, Hypertension } \\ \text { control and associated factors } \\ \text { in Chinese adults. }\end{array} & \begin{array}{l}75,526 \text { records } \\ \text { of 24,410 adults } \\ \text { in 1991-2011 CHNS }\end{array} & \begin{array}{l}\text { Compare effect of } \\ \text { insurance with } \\ \text { non-insurance }\end{array}\end{array}$

Compared with those without medical insurance, hypertensive patients who had medical insurance were more likely to be aware of their hypertensive condition (aOR 1.5, $95 \% \mathrm{Cl} 1.4-1.7$ ), more likely to be medicated for hypertension (aOR $1.7,95 \% \mathrm{Cl}$ 1.5-1.8) and more likely to control their blood pressure within normal range (aOR 1.3, $95 \%$ Cl 1.2-1.4).

Basu et al $2013 \quad$ Social epidemiology of hypertension in middle-income countries determinants of prevalence, diagnosis, treatment, and control in the WHO SAGE study.

Feng et al 2014

Health system strengthening and hypertension awareness, treatment and control:data from the China Health and Retirement Longitudinal Study

Bulletin of the 13,707 individuals World Health of CHARLs

Organization

15050 subject in China of SAGA data

Compare effect of

Chinese Journal 29,411 subjects in of Epidemiology six provinces

Hypertension Control in Communities (HCC): evaluation result of blood pressure

management among hypertensive. Effects of guideline-based rural areas of Guangdong Province.
Chinese Medical 3113 patients with Journal essential hypertension in rural Guangdong

2397 patients in China and Nigeria voluntary insurance with mandatory insurance

Compare the effect of insurance providing costs of outpatient care with those that contributed nothing to this part

Compared with people covered by mandatory insurance, the risk of being undiagnosed was higher (aOR 4.286, $95 \% \mathrm{Cl}$ 1.159-15.84, ten times, $95 \% \mathrm{Cl} 2.1-47.4$ in Chinese cohort), the risk of being untreated was near four times higher (aOR $4.64,95 \% \mathrm{Cl} 1.05-20.46)$, as well as the risk of being uncontrolled (aOR 4.51, $95 \%$ Cl 0.98-20.82) .

Those with insurance that covered the costs of outpatient care were less likely to be unaware of their hypertension (30.1 \% VS $44.4 \%$, aRR: 0.74; $95 \%$ Cl: $0.62-0.88)$, to be untreated for it (38.2\% VS $57.8 \%$, aRR: 0.80 ; $95 \% \mathrm{Cl}: 0.68-0.93)$ and to be not controlling it effectively $(71.0 \%$ VS $81.0 \%$, aRR. 0.90; $95 \%$ Cl: 0.83-1.00), when compared with those in the insurance contributed nothing to outpatient care.

Evaluate the effect of After 1-year intervention, hypertension control guideline-based rate raised from 21.6 to $74.7 \%(P<0.05)$, with an hypertension

management

Compare effect of traditional therapy and the guidelinebased HTN management (the novel therapy) estimated effect of $53.1 \%(95 \%$ Cl 52.4-53.8\%).

After 2 years following up, hypertension treatment and control increased in both groups and the control rate increased more significantly in the guideline-based group (from 31.1 to $37.9 \%$ in the traditional group, and from 26.9 to $64.3 \%$ in the guideline-based group, $P<0.001$ ).

After 12-month following up, the reductions of systolic blood pressure and diastolic blood 
Table 3 Key findings of the eleven most important studies (Continued)

\begin{tabular}{|c|c|c|c|c|c|c|c|}
\hline & & & $\begin{array}{l}\text { control in primary care in } \\
\text { low-resource settings } \\
\text { a cluster-randomized trial }\end{array}$ & $\begin{array}{l}\text { Bulletin of the } \\
\text { World Health } \\
\text { Organization }\end{array}$ & & $\begin{array}{l}\text { Evaluate the effect } \\
\text { of WHO CVD risk } \\
\text { management package }\end{array}$ & $\begin{array}{l}\text { pressure were significantly greater in the } \\
\text { intervention group, with the marginal effects } \\
\text { size of } 3.86 \text { and } 1.53 \mathrm{mmHg} \text { in China }(-13.28 \\
\text { vs }-9.42 \mathrm{mmHg},-6.07 \text { vs }-4.54 \mathrm{mmHg}, P<0.001)\end{array}$ \\
\hline \multirow[t]{4}{*}{$\begin{array}{l}\text { Health Service } \\
\text { delivery }\end{array}$} & Lu et al & 2015 & $\begin{array}{l}\text { Community-based interventions } \\
\text { in hypertensive patients a } \\
\text { comparison of three health } \\
\text { education strategies }\end{array}$ & $\begin{array}{l}\text { BMC Public } \\
\text { Health }\end{array}$ & $\begin{array}{l}360 \text { participants } \\
\text { in Guangdong }\end{array}$ & $\begin{array}{l}\text { Compare three health } \\
\text { education strategies } \\
\text { including self-learning, } \\
\text { monthly lecture and } \\
\text { interactive education } \\
\text { workshop. }\end{array}$ & $\begin{array}{l}\text { Compared with self-learning, after the } 2-y \\
\text { intervention, the likelihood of normalized } \\
\text { BP was greater in lecture group (from } 41.2 \\
\text { to } 63.2 \%, p<0.001 \text {, aOR } 2.37,95 \% \mathrm{Cl} \\
1.26-4.47 \text { ) and interactive workshop group } \\
\text { (from } 40.2 \text { to } 86.3 \%, p<0.001 \text {, aOR } 14.66 \text {, } \\
95 \% \mathrm{Cl} 6.59-32.62 \text { and } 2.37 \text { ). }\end{array}$ \\
\hline & Yun et al & 2014 & $\begin{array}{l}\text { Effectiveness of a Community- } \\
\text { Based Individualized Lifestyle } \\
\text { Intervention Among Older } \\
\text { Adults With Diabetes } \\
\text { and Hypertension, Tianjin, } \\
\text { China, 2008-2009 }\end{array}$ & $\begin{array}{l}\text { Prev Chronic } \\
\text { Dis }\end{array}$ & $\begin{array}{l}213 \text { participants of } \\
\text { five local community } \\
\text { health clinics } \\
\text { in Tianjin }\end{array}$ & $\begin{array}{l}\text { Evaluate the effect } \\
\text { of Zhiji management }\end{array}$ & $\begin{array}{l}\text { Systolic blood pressure and diastolic blood } \\
\text { pressure decreased significantly by } 10.9 \text { and } \\
4.0 \mathrm{mmHg} \text { in the treatment group }(P<0.001) \text {. }\end{array}$ \\
\hline & Niu et al & 2014 & $\begin{array}{l}\text { Differences and determinants } \\
\text { in access to essential public } \\
\text { health services in China a } \\
\text { case study with hypertension } \\
\text { people and under-sixes } \\
\text { as target population. }\end{array}$ & $\begin{array}{l}\text { Chin Med } \\
J \text { (Engl) }\end{array}$ & $\begin{array}{l}1505 \text { hypertensive } \\
\text { patients }\end{array}$ & $\begin{array}{l}\text { Evaluate the effect of } \\
\text { accessibility }\end{array}$ & $\begin{array}{l}\text { The control rate in those who lived less } \\
\text { than } 5 \text { min away from the nearest health } \\
\text { institution was } 39.3 \% \text {, slightly higher } \\
\text { than the rate for those who lived more } \\
\text { than } 20 \text { min away, which was } 35.8 \% \\
\text { (aOR } 1.03,95 \% \text { Cl 0.60-1.79). }\end{array}$ \\
\hline & Gu et al & 2015 & $\begin{array}{l}\text { The Cost-Effectiveness of } \\
\text { Low-Cost Essential } \\
\text { Antihypertensive Medicines } \\
\text { for Hypertension } \\
\text { Control in China: A } \\
\text { Modelling Study }\end{array}$ & PLOS Medicine & & $\begin{array}{l}\text { Evaluate the cost- } \\
\text { effectiveness } \\
\text { of different treating } \\
\text { strategy }\end{array}$ & $\begin{array}{l}\text { Treating all hypertensives for primary } \\
\text { and secondary prevention to goal } \\
\text { of }<140 / 90 \text { if age } 35-64 \text { years, goal } \\
\text { of }<150 / 90 \text { if age } 65 \text { could prevent } \\
\text { about } 800,000 \text { cardiovascular disease } \\
\text { events annually ( } 95 \% \text { Cl } 0.6-1.0 \text { million) } \\
\text { and was borderline cost-effective } \\
\text { incremental to treating only cardiovascular } \\
\text { disease and stage two patients ( } 2015 \\
\text { Int } \$ 13,000 \text { per QALY gained [ } 95 \% \mathrm{Cl} \text {, } \\
\text { Int } \$ 10,000 \text { to Int } \$ 18,000] \text { ) }\end{array}$ \\
\hline
\end{tabular}




\section{Health governance}

Only one cross-sectional study explored the effect of health governance on hypertension management outcomes. Wong et al conducted the study in six cities of southern China in the year 2010 [33]. Using data covering about 1830 hypertensive patients, the study compared performance of hypertension management among three different organized community health care centers (CHCs). The authors found that compared with hospitalfunded $\mathrm{CHCs}$, government-funded $\mathrm{CHCs}$ had significantly lower prescriptions of anti-hypertensive pharmaceutics with an aOR of 0.46 (95\% CI 0.33-0.66). However hypertension subjects managed by government-funded $\mathrm{CHCs}$ were more likely to control their blood pressure adequately (aOR 1.63, $95 \%$ CI 1.16-2.29), while those in privately-funded $\mathrm{CHCs}$ were less likely to achieve optimal blood pressure control (aOR 0.15, 95 \% CI 0.07-0.31). The authors interpreted that government-funded primary care were less incentivized to prescribe antihypertensive agents, and were more willing to implement policies and guidelines. Since the study was institutional based which only recruited hypertensive patients for study, differences in hypertension awareness for population managed by these three types of $\mathrm{CHCs}$ were not analyzed.

\section{Health financing}

All the three papers were based on cross-sectional designs and analyzed the effects of health insurance coverage on the outcomes of hypertension management. The initial attempt was made by Feng et al. [13] in 2014. Using national data from the China Health and Retirement Longitudinal Study (CHARLs), which investigated 17,708 Chinese individuals aged 45 or older, they found that health insurance coverage was the single most important factor associated with good hypertension management in China. As the authors reported, when compared with patients who were members of insurance schemes that cover costs of outpatient care, such as the Urban Employee Basic Medical Insurance Scheme and the Government Insurance Scheme, those who were members of insurance schemes that do not covered costs of outpatient care, like the Urban Resident Basic Medical Insurance Scheme or the New Cooperative Medical Scheme, had nearly $30 \%$ lower awareness (aRR 0.74, $95 \%$ CI $0.62-0.88$ ), $20 \%$ lower treatment (aRR 0.80, $95 \%$ CI $0.68-0.93$ ) and $10 \%$ lower control (aRR 0.90, $95 \% \mathrm{CI}$ 0.83-1.00) rates for their hypertensive disorders. Data from other sources strengthened such associations. For example, using data from the China Health and Nutrition Surveys (CHNS), Guo et al. reported in the year 2015 that hypertensive patients who had medical insurance were more likely to be aware of their hypertensive condition (aOR 1.50, 95 \% CI 1.40-1.70), more likely to be medicated for hypertension (aOR 1.70, 95 \% CI 1.50-1.80) and more likely to control their blood pressure within normal range (aOR 1.30, $95 \%$ CI 1.20-1.40) [22]. And using the SAGE data, Basu et al. found that compared with people covered by mandatory insurance, those covered by voluntary insurance had a ten times greater odds of undiagnosed hypertension (95 \% CI 2.1047.40 ) in the Chinese sample, and the risk of being untreated was near four times higher (aOR 4.64, 95 \% CI 1.05-20.46), as well as the risk of being uncontrolled (aOR 4.51, 95 \% CI 0.98-20.82) [23].

\section{Health information}

The WHO developed a guideline in the World Health Report 2002 using a total risk approach for the prevention of cardiovascular disease [34]. Based on regression models with age, sex, smoking, blood pressure, blood cholesterol, and presence of diabetes as predictors, individuals could be classified into four risk categories of fatal or nonfatal vascular events: very high risk, high risk, moderate risk and low risk. For the individuals of very high or high risk, 3-6 months' following up for risk monitoring are recommended, 6-12 months' following up intervals are suggested for those at moderate risk, whilst no follow up were suggested for low risk individuals other than health education on life style modifications. Following up interventions include regular blood pressure monitoring, lifestyle modification consultation like dietary changes, physical activity and weight control, alcohol consumption control, and anti-hypertensive prescription and adjustment. A cardiovascular risk management package was further developed by the WHO later [35]. Main elements includes i) risk-assessment and risk-management algorithms; lifestyle counseling protocols; anti-hypertensive agents' treatment protocols; referral pathways and follow-up schedules; ii) tools for both providers and patients (training program and manual; self-management tools; personal health records; and tools for improving adherence).

Following the WHO, China already developed its own guidelines called Chinese Guidelines for the Management of Hypertension [36]. The Guideline firstly described the current situation of the increasing prevalence and relatively ineffective management of hypertension in China. And it then recommended a combination of primary, secondary and tertiary prevention, where two strategies were proposed: population strategy and high risk strategy. The population strategy emphasized a healthy lifestyle, health education, community involvement for all citizens; whilst the high risk strategy recommended health examinations and health management targeting high risk population. As for all the hypertensive patients, the Guideline suggested the establishment of personal health record, and community health promotions to improve outpatient attendances 
and self-management. Since the Chinese guideline was developed based on the WHO's, they were similar in most parts. However, none risk assessment algorithms were presented in the Chinese guideline.

Three included articles assessed the effects in carrying out such guidelines for hypertension management. A trial by Wang et al. carried out in six provinces in China evaluated the effect of guideline-based blood pressure management [37]. The authors reported that after 1-year intervention, hypertension control rate raised from 21.6 to $74.7 \%(P<0.05)$, with an estimated effect of $53.1 \%$ (95\% CI 52.4-53.8\%). Li et al. did a quasi-experiment in the year 2015 comparing the performance between traditional therapy and the guideline-based hypertension management [38]. The traditional therapy referred to health education on what is hypertension, the potential consequence of hypertension and the risk factors associated with hypertension. After 2 years follow up, the study showed that hypertension treatment and control increased in both groups and the control rate increased more significantly in the guideline-based group (from 31.1 to $37.9 \%$ in the traditional group, and from 26.9 to $64.3 \%$ in the guideline-based group, $P<0.001)$. Mendis et al. conducted a clustered randomized control trial based on WHO cerebrovascular disease risk management package in China and Nigeria in the year 2006 [7]. The study indicated that introduction of the package resulted in a significant increase in the prescribing of anti-hypertensive agents and after 12-month follow up, the reductions of systolic blood pressure and diastolic blood pressure were significantly greater in the intervention group, with the marginal effects size of 3.86 and $1.53 \mathrm{mmHg}$ in China $(-13.28$ vs $-9.42 \mathrm{mmHg},-6.07$ vs $-4.54 \mathrm{mmHg}, P<0.001)$.

\section{Health service delivery}

Four researches explicated various mechanisms on how to deliver essential health care service to the hypertensive patients properly and efficiently, among which two randomized control trials investigated strategies to improve the efficacy of lifestyle health education practices; and the other two cross-sectional studies, one analyzed the association between access to health care and hypertension management outcomes. And the other one is a modelbased study evaluating the gains and cost-effectiveness of implementing total patients management as compare to severe case management.

Lu et al. conducted a community based health education trial in Dongguan City, Guangdong province during 2011-2013 [39]. With a small sample size of 347, the authors compared the performance of three health education strategies in improving the outcomes of hypertension management. It found that regular use of medications to treat hypertension was more frequent when monthly interactive education workshop and monthly didactic lecture were used (92.3 and $79.8 \%$ vs $50.9 \%, P=0.001$ ), as compared to the self-learning material distribution strategy. And hypertension control rates were also higher when the two more active health education strategies were used (aOR 14.66, 95 \% CI 6.59-32.62 and 2.37, 95 \% CI 1.26-4.47 respectively). The other study conducted by Yun et al. in Tianjin in 2014 corroborated the positive effects for such active education strategy [40]. Based on a "Zhiji management", i.e. health education by friends, it found systolic blood pressure and diastolic blood pressure decreased significantly by 10.9 and $4.0 \mathrm{mmHg}$ in the treatment group $(P<0.001)$.

Niu et al. assessed the association of accessibility of essential public health services and good hypertension management [41]. It was reported that the distance between the nearest primary health care institution and the homes of patients could influence the likelihood to the hypertensives to achieve optimal blood pressure control, where the control rate in those who lived less than 5 min away from the nearest health institution was $39.3 \%$, slightly higher than the rate for those who lived more than 20 min away, which was $35.8 \%$ (aOR 1.03, 95 \% CI 0.60-1.79).

$\mathrm{Gu}$ et al. conducted a model-based cost-effectiveness analysis comparing total patients management with severe case management. It was found that treating all hypertensives for primary and secondary prevention to goal of $<140 / 90$ if age $35-64$ years, goal of $<150 / 90$ if age $\geq 65$ could prevent about 800,000 cardiovascular disease events annually (95\% CI $0.6-1.0$ million) and was borderline cost-effective incremental to treating only cardiovascular disease and stage two patients (2015 Int $\$ 13,000$ per QALY gained [95 \% CI, Int $\$ 10,000$ to Int $\$ 18,000])$ [42]. That means the total patients management strategy seems to be more effective with slightly incremental cost than severe case management.

\section{Conclusions}

The prevalence of hypertension has been rising rapidly in China. Our systematical search found quite a few epidemiological researches on the prevalence, awareness, treatment and control of hypertension in China which had been intentionally described. It was reported that the management of hypertension in China is a detection problem rather than treatment problem. And the efforts of public health community were also abundant, mainly investing in community based health promotion programs. However, less attentions were paid by the Chinese researchers to investigate in the mechanisms that linked various health system factors to the outcome and impact of hypertension management in China, while no evidence was found to clearly link between health system factors and hypertension incidence or its population impact, such as morbidity, mortality, quality of life and financial 
burden. For system factors, we identified no evidence on the association between human resources for health and physical resources for health and hypertension management. While we only identified paucity of evidence with limited study quality, showing that the improving health system governance, health finance, health information, and health service delivery can make a difference on hypertension management since good governance in community health centers, more generous health insurance covering outpatient care, available guideline directing management and interactive health education strategy as well as total patients management were reported to be associated with good hypertension management. It is therefore strongly called for joint efforts from health system and epidemiological researchers to extend knowledge in this area.

\section{Additional file}

Additional file 1: Appendix 1. (XLSX $408 \mathrm{~kb})$

\section{Abbreviations}

CHARLs: China Health and Retirement Longitudinal Study; CHCs: Health care centers; CHNS: China Health and Nutrition Surveys; CNKI: China National Knowledge Infrastructure; CVDs: Cardiovascular diseases; LMICs: Low-and middle-income countries; NCDs: Non-communicable diseases; SAGE: Study on Global Aging and Adult Health; WHO: World Health Organization

\section{Acknowledgements}

None.

\section{Funding}

National Natural Science Foundation of China (71303010); China National Natural Science Foundation Excellent Young Scientist Program(71422009).

\section{Availability of data and materials}

The data that support the findings of this review are available in Additional file 1: Appendix 1.

\section{Authors' contributions}

XLF conceived the paper. KH led the systematic review and data extraction under XLF's supervision. YTS and YHH participated in the systematic review and data extraction. KH and XLF wrote this paper. All authors read and approved the final manuscript.

\section{Consent for publication}

Not applicable.

\section{Competing interests}

The authors declare that they have no competing interests.

\section{Ethics approval and consent to participate}

Not applicable.

Received: 16 April 2016 Accepted: 9 September 2016

Published online: 21 September 2016

\section{References}

1. WHO. Global status report on noncommunicable diseases 2010. Geneva: World Health Organization; 2011.

2. Lozano R, et al. Global and regional mortality from 235 causes of death for 20 age groups in 1990 and 2010: a systematic analysis for the Global Burden of Disease Study 2010. Lancet. 2012;380(9859):2095-128.
3. UN General Assembly. Political declaration of the high-level meeting of the General Assembly on the prevention and control of non-communicable diseases. New York: United Nations; 2011.

4. Lewington $\mathrm{S}$, et al. Age-specific relevance of usual blood pressure to vascular mortality: a meta-analysis of individual data for one million adults in 61 prospective studies. Lancet. 2002;360(9349):1903-13.

5. Lim SS, et al. A comparative risk assessment of burden of disease and injury attributable to 67 risk factors and risk factor clusters in 21 regions, 19902010: a systematic analysis for the Global Burden of Disease Study 2010. Lancet. 2012;380(9859):2224-60.

6. WHO. A global brief on hypertension-silent killer, global public health crisis. 2013.

7. Mendis S, et al. Cardiovascular risk management and its impact on hypertension control in primary care in low-resource settings: a cluster-randomized trial. Bull World Health Organ. 2010;88(6):412-9.

8. Ibrahim MM, Damasceno A. Hypertension in developing countries. Lancet. 2012;380(9841):611-9.

9. James PA, et al. 2014 evidence-based guideline for the management of high blood pressure in adults: report from the panel members appointed to the Eighth Joint National Committee (JNC 8). JAMA. 2014;311(5):507-20.

10. Xu W, et al. Optimal systolic blood pressure target, time to intensification, and time to follow-up in treatment of hypertension: population based retrospective cohort study. BMJ. 2015;350:h158.

11. WHO. Everybody's business: Strengthening health systems to improve health outcomes: WHO's framework for action. Geneva: World Health Organization; 2007.

12. Maimaris W, et al. The Influence of Health Systems on Hypertension Awareness, Treatment, and Control: A Systematic Literature Review. PLoS Med. 2013;10(7):e1001490.

13. Feng $X L$, Pang $M$, Beard J. Health system strengthening and hypertension awareness, treatment and control: data from the China Health and Retirement Longitudinal Study. Bull World Health Organ. 2014;92(1):29-41.

14. He J, et al. Premature deaths attributable to blood pressure in China: a prospective cohort study. Lancet. 2009;374(9703):1765-72.

15. WHO. Monitoring the building blocks of health systems. 2010.

16. WHO. Checklist and Indicators for Monitoring Progress in the Development of IHR Core Capacities in States Parties. 2005.

17. Gu D, et al. Prevalence, Awareness, Treatment, and Control of Hypertension in China. Hypertension. 2002;40(6):920-7.

18. Reynolds $\mathrm{K}$, et al. Geographic variations in the prevalence, awareness, treatment and control of hypertension in China. J Hypertens. 2003;21 (7):1273-81.

19. Dong $\mathrm{G}$, et al. Prevalence, awareness, treatment, and control of hypertension in rural adults from Liaoning Province, northeast China. Hypertens Res. 2007;30(10):951-8.

20. Li H, et al. Prevalence, awareness, treatment, and control of hypertension in rural China: results from Shandong Province. J Hypertens. 2010;28(3):432-8.

21. Li D, et al. Hypertension burden and control in mainland China: Analysis of nationwide data 2003-2012. Int J Cardiol. 2015;184:637-44.

22. Guo J, et al. The dynamics of hypertension prevalence, awareness, treatment, control and associated factors in Chinese adults. J Hypertens. 2015;33(8):1688-96.

23. Basu S, Millett C. Social Epidemiology of Hypertension in Middle-Income Countries: Determinants of Prevalence, Diagnosis, Treatment, and Control in the WHO SAGE Study. Hypertension. 2013;62(1):18-26.

24. Lloyd-Sherlock P, et al. Hypertension among older adults in low- and middle-income countries: prevalence, awareness and control. Int J Epidemiol. 2014;43(1):116-28.

25. Wang J, et al. Prevalence, awareness, treatment, and control of hypertension in China: results from a national survey. Am J Hypertens. 2014;27(11):1355-61.

26. Bi Z, et al. Hypertension prevalence, awareness, treatment, and control and sodium intake in Shandong Province, China: baseline results from Shandong-Ministry of Health Action on Salt Reduction and Hypertension (SMASH), 2011. Prev Chronic Dis. 2014;11:E88.

27. Wang $\mathrm{H}$, et al. Factors associated with prevalence, awareness, treatment and control of hypertension among adults in Southern China: a community-based, cross-sectional survey. PLoS One. 2013;8(5):e62469.

28. Zhang J, et al. Prevalence, awareness, medication, control, and risk factors associated with hypertension in Bai ethnic group in rural China: the Yunnan Minority Eye Study. PLoS One. 2013;8(8):e70886.

29. Zhao Y, et al. Trends in population blood pressure and prevalence, awareness, treatment, and control of hypertension among middle-aged and 
older adults in a rural area of Northwest China from 1982 to 2010. PLoS One. 2013;8(4):e61779.

30. Hou Z, Meng Q, Zhang Y. Hypertension Prevalence, Awareness, Treatment, and Control Following China's Healthcare Reform. Am J Hypertens. 2015;29(4):428-31.

31. Zeng $X Y$, et al. The study of management of hypertensive people aged 35 and over in communities in China. Zhonghua Yu Fang Yi Xue Za Zhi. 2013; 47(11):1014-9.

32. Department, H. National essential public health services (2009). 2009. p. 1.

33. Wong MCS. Performance Comparison among the Major Healthcare Financing Systems in Six Cities of the Pearl River Delta Region, Mainland China. PLOS ONE. 2012;7(9):1-3.

34. WHO. Prevention of Cardiovascular Disease. 2007.

35. WHO. Integrated Management of Cardiovascular Risk. 2002.

36. Liu L. Guidelines for prevention and treatment of hypertension in China. Chin J Hypertens. 2011;19(8):701-43.

37. Wang Z, Wang X, Zhang L. Community hypertension control: evaluation of the effect of blood pressure management. Chin J Epidemiol. 2010;31(1):1-4.

38. Li G. Effects of Guideline-based Hypertension Management in Rural Areas of Guangdong Province. Chin Med J. 2015;128(6):799-803.

39. Lu C, et al. Community-based interventions in hypertensive patients: a comparison of three health education strategies. BMC Public Health. 2015;15(1):33.

40. Yun R, Yan LL. Effectiveness of a Community-Based Individualized Lifestyle Intervention Among Older Adults With Diabetes and Hypertension, Tianjin China, 2008-2009. Prev Chronic Dis. 2014;11:1-7.

41. Niu H, et al. Differences and determinants in access to essential public health services in China: a case study with hypertension people and under-sixes as target population. Chin Med J (Engl). 2014;127(9):1626-32

42. Gu D, et al. The Cost-Effectiveness of Low-Cost Essential Antihypertensive Medicines for Hypertension Control in China: A Modelling Study. PLoS Med. 2015;12(8):e1001860.

\section{Submit your next manuscript to BioMed Central and we will help you at every step:}

- We accept pre-submission inquiries

- Our selector tool helps you to find the most relevant journal

- We provide round the clock customer support

- Convenient online submission

- Thorough peer review

- Inclusion in PubMed and all major indexing services

- Maximum visibility for your research

Submit your manuscript at www.biomedcentral.com/submit

) Biomed Central 